\title{
Remarks to "on strong intuitionistic fuzzy metrics"
}

\section{Valentín Gregori*, Almanzor Sapena}

Universitat Politècnica de València, Instituto Universitario de Matemática Pura y Aplicada, Campus de Gandia, Paranimf 1, 46730, Grau de Gandia, Spain.

\author{
Communicated by C. Alaca
}

\begin{abstract}
According to the concept of strong (non-Archimedean) fuzzy metric, in the sense of George and Veeramani, Efe and Yigit have introduced, and studied, the concept of strong intuitionistic fuzzy metric [H. Efe, E. Yigit, J. Nonlinear Sci. Appl., 9 (2016), 4016-4038]. In this note we show that all results obtained by the authors are immediate consequences of known results in fuzzy metric setting and a few simple results that we will introduce.
\end{abstract}

Keywords: t-norm, t-conorm, (strong) fuzzy metric, (strong) intuitionistic fuzzy metric.

2010 MSC: 54A40, 54E35.

(C)2018 All rights reserved.

\section{Introduction and preliminaries}

Several concepts of fuzzy metrics have been given, from different points of view, in fuzzy setting. Here we deal with the concept of fuzzy metric given by George and Veeramani [9], which is a modification of the one due to Kramosil and Michalek in [11] where they extended the concept of Menger space to fuzzy setting.

Gregori et al. defined the concept of strong (non-Archimedean) fuzzy metric in [4] where they studied the completion of this type of fuzzy metrics. Further studies on strong fuzzy metrics can be seen in $[3,5,13]$.

Park introduced and discussed in [12] a notion of intuitionistic fuzzy metric which is based both on the idea of intuitionistic fuzzy set due to Atanassov [1] and the concept of fuzzy metric due to George and Veeramani. Recently, Efe and Yigit, [2], have introduced and studied the concept of strong intuitionistic fuzzy metric based on the concept of strong fuzzy metric.

In [8] the authors advised that many possible constructions in the realm of intuitionistic fuzzy metric spaces can be directly obtained from the fuzzy case. In this way, we think that the notion of strong intuitionistic fuzzy metric has little margin in light of Proposition 4.2.

\footnotetext{
${ }^{*}$ Corresponding author

Email addresses: vgregori@mat.upv.es (Valentín Gregori), alsapie@mat.upv.es (Almanzor Sapena)

doi: $10.22436 /$ jnsa.011.02.12
}

Received: 2016-09-26 Revised: 2017-05-26 Accepted: 2017-05-26 
In this paper, after preliminaries and reminders on strong fuzzy metrics, we prove in Section 3 and Section 4 that all results obtained in [2] are immediate consequences of known results in (strong) fuzzy metrics and a few of simple results which we will introduce. In addition, we also correct some assertions found in the mentioned paper and we have updated some results related to the topology deduced from an intuitionistic fuzzy metric space.

In the following $\mathbb{N}, \mathbb{R}$, and $\mathbb{R}^{+}$will denote the sets of positive integers, real numbers, and positive real numbers, respectively.

Recall [14] that a continuous t-norm is a binary operation $*:[0,1] \rightarrow[0,1]$ which satisfies the following conditions: (i) $*$ is associative and commutative; (ii) $*$ is continuous; (iii) $a * 1=a$ for every $a \in[0,1]$; (iv) $a * b \leqslant c * d$ whenever $a \leqslant c$ and $b \leqslant d$, and $a, b, c, d \in[0,1]$.

Similarly, a continuous t-conorm is a binary operation: $\diamond:[0,1] \rightarrow[0,1]$ which satisfies the following conditions: (i) $\diamond$ is associative and commutative; (ii) $\diamond$ is continuous; (iii) $a \diamond 0=a$ for every $a \in[0,1]$; (iv) $a \diamond b \leqslant c \diamond d$ whenever $a \leqslant c$ and $b \leqslant d$, and $a, b, c, d \in[0,1]$.

Given a $\mathrm{t}$-norm $*$, the $\mathrm{t}$-conorm $\diamond$ given by $\mathrm{a} \diamond \mathrm{b}=1-((1-\mathrm{a}) *(1-\mathrm{b}))$ is the $\mathrm{t}$-conorm associated to $*$. In this case it is also satisfied that $a * b=1-((1-a) \diamond(1-b))$. So, it is said that $*$ and $\diamond$ are associated. The three continuous t-norms mostly used in fuzzy setting are the t-norm minimum $(\wedge)$ given by $a \wedge$ $\mathrm{b}=\min \{\mathrm{a}, \mathrm{b}\}$, the usual product $(\cdot)$ and the Lukasievicz $\mathrm{t}$-norm $(\mathfrak{L})$ given $b y \mathfrak{a} \mathfrak{b}=\max \{0, \mathrm{a}+\mathrm{b}-1\}$. The $t$-conorms $\diamond$ associated to the mentioned $t$-norms are $a \diamond b=\max \{a, b\}, a \diamond b=a+b-a b$ and $\mathrm{a} \diamond \mathrm{b}=\min \{1, \mathrm{a}+\mathrm{b}\}$, respectively.

Recall that $x \wedge y \geqslant x \cdot y \geqslant x \mathfrak{L} y$ for all $x, y \in[0,1]$. We denote it, briefly, by $\wedge \geqslant \cdot \geqslant \mathfrak{L}$. Also it is well known that $\min \leqslant *$ for all $\mathrm{t}$-norm $*$.

In order to simplify the presentation of this paper, in the following $*$ will denote a continuous $\mathrm{t}$ norm and $*^{\prime}$ its associated continuous t-conorm. Also, $\diamond$ will denote a continuous $\mathrm{t}$-conorm and $\diamond^{\prime}$ its associated continuous t-norm. Then, by the above definitions it is easy to obtain the following proposition.

\section{Proposition 1.1.}

(i) $\left(*^{\prime}\right)^{\prime}=*$ and $\left(\diamond^{\prime}\right)^{\prime}=\diamond$;

(ii) $\mathrm{a} * \mathrm{~b} \leqslant \mathrm{c}$ if and only if $(1-\mathrm{a}) *^{\prime}(1-\mathrm{b}) \geqslant 1-\mathrm{c}$;

(iii) $a \diamond b \geqslant c$ if and only if $(1-a) \diamond^{\prime}(1-b) \leqslant 1-c$;

(iv) if $*_{1}$ is a $\mathrm{t}$-norm with $* \leqslant *_{1}$, then $*^{\prime} \geqslant *_{1}^{\prime}$;

(v) if $\diamond_{1}$ is a t-conorm with $\diamond \leqslant \diamond_{1}$, then $\diamond^{\prime} \geqslant \diamond_{1}^{\prime}$.

Park introduced in [12] the following notion (in which we use a double terminology for easy the reading of the paper).

Definition 1.2. An intuitionistic fuzzy metric space is a 5-tuple $(X, M, N, *, \diamond)$ such that $X$ is a (nonempty) set, $*$ is a continuous t-norm, $\diamond$ is a continuous $t$-conorm, and $M, N$ are fuzzy sets on $X \times X \times(0, \infty)$ satisfying the following conditions, for all $x, y, z \in X, s, t>0$ :

$($ IFM-1) $M(x, y, t)+N(x, y, t) \leqslant 1$;

(GV1) (IFM-2) $M(x, y, t)>0$;

(GV2) (IFM-3) $M(x, y, t)=1$ if and only if $x=y$;

(GV3) (IFM-4) $M(x, y, t)=M(y, x, t)$;

$(\mathrm{GV} 4)(\mathrm{IFM}-5) M(x, y, t) * M(y, z, s) \leqslant M(x, z, t+s)$;

(GV5) (IFM-6) $M_{x, y}: \mathbb{R}^{+} \rightarrow(0,1]$ is a continuous function where $M_{x y}(t)=M(x, y, t)$;

(IFM-7) $N(x, y, t)<1$;

(IFM-8) $N(x, y, t)=0$ if and only if $x=y$; 
(IFM-9) $N(x, y, t)=N(y, x, t)$;

$($ IFM-10) $N(x, y, t) \diamond N(y, z, s) \geqslant N(x, z, t+s)$;

$\left(\right.$ IFM-11) $N_{x, y}: \mathbb{R}^{+} \rightarrow[0,1)$ is a continuous function where $N_{x y}(t)=N(x, y, t)$;

If $(X, M, N, *, \diamond)$ is an intuitionistic fuzzy metric space (IFMS for shorten) we will say that $(M, N, *, \diamond)$ is an intuitionistic fuzzy metric on $X$, or simply $(M, N)$ is an IFM, if confusion is not possible or not further information is needed. This terminology will be used for the rest of intuitionistic (fuzzy) notions without explicit mention.

Definition 1.3 ([9]). A fuzzy metric space is an ordered triple $(X, M, *)$ such that $X$ is a (nonempty) set, $*$ is a continuous $t$-norm, and $M$ is a fuzzy set on $X \times X \times \mathbb{R}^{+}$satisfying the five above conditions (GV1)-(GV5) for all $x, y, z \in X, s, t>0$.

It is clear that if $(X, M, N, *, \diamond)$ is an IFMS, then $(X, M, *)$ is a fuzzy metric space. Conversely, by (ii) of Proposition 1.1, we have the following proposition.

Proposition $1.4([8, \operatorname{Remark} 1$ (B)] and $[2, \operatorname{Remark} 1.5$ (i) $])$. If $(X, M, *)$ is a fuzzy metric space, then $(X, M, 1-$ $\left.\mathrm{M}, *, *^{\prime}\right)$ is an IFMS.

The following proposition is obvious.

Proposition 1.5. Let $*$ and $\star$ be two continuous $\mathrm{t}$-norms with $\star \leqslant *$. If $(\mathrm{X}, \mathrm{M}, *)$ is a fuzzy metric space, then $(\mathrm{X}, \mathrm{M}, \star)$ is a fuzzy metric space.

If $(X, d)$ is a metric space, then $\left(X, M_{d}, *\right)$ is a fuzzy metric space called standard fuzzy metric space induced by $(X, d)$, where $M_{d}(x, y, t)=\frac{t}{t+d(x, y)}$ and $*$ is the usual product. Then, by Proposition 1.4 $\left(X, M_{d}, 1-M_{d}, *, *^{\prime}\right)$ is an IFMS called standard in [2] (the same is true if $\left.*=\wedge\right)$.

George and Veeramani proved in [9] that every fuzzy metric $M$ on $X$ generates a topology $\tau_{M}$ on $X$, which has as a base the family of open sets of the form $\left\{B_{M}(x, \varepsilon, t): x \in X, 0<\varepsilon<1, t>0\right\}$, where $B_{M}(x, \varepsilon, t)=\{y \in X: M(x, y, t)>1-\varepsilon\}$ for all $\left.x \in X, \varepsilon \in\right] 0,1\left[\right.$ and $t>0$. It is also said that $\tau_{M}$ is the topology induced by $M$ or deduced from $M$. This topology is metrizable [6, 10]. It is well known that a sequence $\left\{x_{n}\right\}$ converges to $x_{0}$ in $\tau_{M}$ if and only if $\lim _{n} M\left(x_{n}, x_{0}, t\right)=1$ for all $t>0$.

Park proved in [12] that each IFM $(M, N)$ on $X$ generates a topology $\tau_{(M, N)}$ on $X$. Later, in $[8$, Theorem $2]$, the authors proved the following result.

Proposition 1.6. Let $(X, M, N, *, \diamond)$ be an IFMS. Then $\tau_{(M, N)}$ coincides with $\tau_{M}$. (Consequently, $\tau_{(M, N)}$ is metrizable).

Definition 1.7. A sequence $\left\{x_{n}\right\}$ in a fuzzy metric space $(X, M, *)$ is said to be Cauchy if for each $\left.\epsilon \in\right] 0,1[$ and each $t>0$ there exists $n_{0} \in \mathbb{N}$ such that $M\left(x_{n}, x_{m}, t\right)>1-\epsilon$ for all $n, m \geqslant n_{0}$ or, equivalently, $\lim _{n, m} M\left(x_{n}, x_{m}, t\right)=1$ for all $t>0 . X$ is said to be complete if every Cauchy sequence in $X$ is convergent with respect to $\tau_{M}$. In such a case $M$ is also said to be complete.

The following is a well-known result.

Proposition 1.8. The function $\mathrm{M}_{x y}$ in axiom (GV5) is non-decreasing for all $x, y \in X$.

Definition 1.9 ([7]). A fuzzy metric $M$ on $X$ is said to be stationary if $M$ does not depend on $t$, i.e., if for each $x, y \in X$, the function $M_{x, y}(t)=M(x, y, t)$ is constant. In this case we write $M(x, y)$ instead of $M(x, y, t)$. According to this definition, an $\operatorname{IFM}(M, N)$ is called stationary [2] if $M$ and $N$ do not depend on $t$.

The following result will be useful in the next sections. 
Proposition $1.10([8,12])$. Let $(X, M, N, *, \diamond)$ be an IFMS. Then the pair $\left(1-\mathrm{N}, \diamond^{\prime}\right)$ is a fuzzy metric on $X$, where $1-N$ is defined on $X^{2} \times \mathbb{R}^{+}$by $(1-N)(x, y, t)=1-N(x, y, t)$.

An immediate consequence is the following.

Proposition 1.11. Let $(X, M, N, *, \diamond)$ be an IFMS. Then

(i) the function $\mathrm{N}_{\mathrm{xy}}$ in axiom (IFM-11) is non-increasing for all $x, y \in X$;

(ii) $\left(\mathrm{X}, 1-\mathrm{N}, \mathrm{N}, \diamond^{\prime}, \diamond\right)$ is an IFMS.

\section{Strong fuzzy metrics (a brief reminder)}

The following notion can be found in $[4,13]$.

Definition 2.1. Let $(X, M, *)$ be a fuzzy metric space. The fuzzy metric $M$ is said to be strong (nonArchimedean) if it satisfies for each $x, y, z \in X$ and each $t>0$

$\left(\mathrm{GV}^{\prime}\right) M(x, z, t) \geqslant M(x, y, t) * M(y, z, t)$.

As a particular case, if $(M, \wedge)$ is strong, we obtain the notion of fuzzy ultrametric $(M(x, z, t) \geqslant$ $M(x, y, t) \wedge M(y, z, t))$.

Remark 2.2. In general, Axiom (GV4') cannot replace (GV4) in order to obtain a fuzzy metric. In fact, in [13, Example 8] it is shown a fuzzy set $M$ on $X^{2} \times \mathbb{R}^{+}$satisfying (GV1)-(GV3), (GV4'), and (GV5) which does not satisfy (GV4). Nevertheless the authors stated the following result.

Proposition 2.3. If we replace (GV4) by (GV4') and we demand in (GV5) that $\mathrm{M}_{\mathrm{xy}}$ be a non-decreasing continuous function, then $\mathrm{M}$ is a strong fuzzy metric.

Strong fuzzy metrics satisfy the following results.

Proposition 2.4 ([13]). Let $(M, *)$ be a non-stationary fuzzy metric on $\mathrm{X}$. Then:

(i) $(M, *)$ is strong if and only if $\left(M_{t}, *\right)$ is a stationary fuzzy on $X$ for each $t>0$ (where $M_{t}$ is the fuzzy set on $\mathrm{X}^{2} \times \mathbb{R}^{+}$given by $\mathrm{M}_{\mathrm{t}}(\mathrm{x}, \mathrm{y})=\mathrm{M}(\mathrm{x}, \mathrm{y}, \mathrm{t})$ );

(ii) further, if $(M, *)$ is strong, then $\tau_{M}=\bigvee\left\{\tau_{M_{t}}: t \in \mathbb{R}^{+}\right\}$.

$\left\{M_{t}: t \in \mathbb{R}^{+}\right\}$is called the family of stationary fuzzy metrics deduced from $M$. It is easy to verify that $M_{t} \succ M_{t^{\prime}}$ whenever $t<t^{\prime}$.

Proposition 2.5 ([13]). Let $\left\{\left(M_{t}, *\right): t \in \mathbb{R}^{+}\right\}$be a family of stationary fuzzy metrics on $\mathrm{X}$. The function $\mathrm{M}$ on $\mathrm{X}^{2} \times \mathbb{R}^{+}$defined by $\mathrm{M}(\mathrm{x}, \mathrm{y}, \mathrm{t})=\mathrm{M}_{\mathrm{t}}(\mathrm{x}, \mathrm{y})$ is a strong fuzzy metric on $\mathrm{X}$ when considering the $\mathrm{t}$-norm $*$, if and only if $\left\{\left(\mathrm{M}_{\mathrm{t}}, *\right): \mathrm{t} \in \mathbb{R}^{+}\right\}$is an increasing family (i.e., $\mathrm{M}_{\mathrm{t}} \leqslant \mathrm{M}_{\mathrm{t}^{\prime}}$ if $\left.\mathrm{t}<\mathrm{t}^{\prime}\right)$ and the function $\mathrm{M}_{\mathrm{xy}}: \mathbb{R}^{+} \rightarrow \mathbb{R}^{+}$ defined by $M_{x y}(t)=M(x, y, t)$ is a continuous function, for each $x, y \in X$.

Proposition 2.6 ([13]). Let $\left\{\mathrm{M}_{\mathrm{t}}: \mathrm{t} \in \mathbb{R}^{+}\right\}$be the family of stationary fuzzy metrics deduced from the strong fuzzy metric $(M, *)$ on $X$. Then the sequence $\left\{x_{n}\right\}$ in $(X, M, *)$ is Cauchy if and only if $\left\{x_{n}\right\}$ is Cauchy in $\left(X, M_{t}, *\right)$ for each $\mathrm{t}>0$.

Corollary $2.7([13])$. Let $(X, M, *)$ be a strong fuzzy metric space. Then $(X, M, *)$ is complete if and only if $\left(\mathrm{X}, \mathrm{M}_{\mathrm{t}}, *\right)$ is complete.

\section{Intuitionistic fuzzy metric spaces}

Observe that the only relationship between $M$ and $N$ in an IFMS is the condition (IFM-1) in Definition 1.2. So, the following lemma is immediate.

Lemma 3.1. Let $(M, *)$ and $(S, \star)$ be two fuzzy metrics on $X$, with $S \leqslant M$ (that is $S(x, y, t) \leqslant M(x, y, t)$ for all $\mathrm{x}, \mathrm{y} \in \mathrm{X}, \mathrm{t}>0)$. If $(\mathrm{X}, \mathrm{M}, \mathrm{N}, *, \diamond)$ is an IFMS then $(\mathrm{X}, \mathrm{S}, \mathrm{N}, \star, \diamond)$ is an IFMS.

Lemma 3.2. $(X, M, N, *, \diamond)$ is an IFMS if and only if $(M, *)$ and $\left(1-N, \diamond^{\prime}\right)$ are fuzzy metrics on $X$ and $M+N \leqslant$ 1 (i.e., $M(x, y, t)+N(x, y, t) \leqslant 1$ for all $x, y \in X, t>0)$. 
Proof. The direct is obvious from Proposition 1.10. We will see the converse. If $\left(1-\mathrm{N}, \diamond^{\prime}\right)$ is a fuzzy metric then by Proposition $1.4\left(X, 1-N, N, \diamond^{\prime}, \diamond\right)$ is an IFMS. Then, by Lemma 3.1, $(X, M, N, *, \diamond)$ is an IFMS, since $M \leqslant 1-N$.

The reader can easily construct many IFMS by means of an IFM $(M, N, *, \diamond)$ or by means of a fuzzy metric $(M, *)$, combining Proposition 1.4, Proposition 1.10, Lemma 3.1, and Lemma 3.2.

Remark 3.3 (Compare with [2, Example 1.6]). Let $(X, d)$ be a metric space and let $m, k_{1}, k_{2} \in \mathbb{R}^{+}$and let $*$ be the usual product. From Example 4 of [5], $\left(M_{i}, *\right)$ is a fuzzy metric on $X$ where $M_{i}(x, y, t)=$ $\frac{k_{i} \cdot t^{n}}{k_{i} \cdot t^{n}+m \cdot d(x, y)}, i=1,2$. Then, by Proposition 1.4 and Lemma 3.2, $\left(M_{1}, 1-M_{2}, *, *^{\prime}\right)$ is an IFMS on $X$ if and only if $M_{1} \leqslant M_{2}$ if and only if $k_{1} \geqslant k_{2}$.

Remark 3.4 ([2, Example 1.8]). It is well known that $\left(\mathbb{R}^{+}, M, *\right)$ is a stationary fuzzy metric space, where $M(x, y)=\frac{\min \{x, y\}}{\max \{x, y\}}$ and $*$ is the usual product. Then, by Propositions 1.4 and 1.5, and Lemma 3.1, $\left(\mathbb{R}^{+}, M, 1-M, \mathfrak{L}, *^{\prime}\right)$ is an IFMS since $\mathfrak{L} \leqslant *$.

Remark 3.5. [2, Proposition 2.8] is a consequence of Proposition 1.4 applied on the fuzzy metric space given in [5, Example 3].

Remark 3.6 (Compare with [2, Proposition 2.10]). Let $\left(\mathrm{X}, \mathrm{K}, \mathrm{P}, *, *^{\prime}\right)$ be a stationary IFMS where $*$ is the usual product. Let $\varphi: \mathbb{R}^{+} \rightarrow \mathbb{R}^{+}$be a continuous increasing function and let $M, N$ be two fuzzy sets on $\mathrm{X}^{2} \times \mathbb{R}^{+}$defined by

$$
M(x, y, t)=\frac{\varphi(t)}{\varphi(t)+1-K(x, y)}, N(x, y, t)=\frac{P(x, y)}{\varphi(t)+P(x, y)} .
$$

By Proposition 1.10 and (i) of Proposition $1.1,(\mathrm{X}, 1-\mathrm{P}, *)$ is a stationary fuzzy metric space. By [5, Example 17],

$$
\frac{\varphi(t)}{\varphi(t)+1-(1-P(x, y))}=\frac{\varphi(t)}{\varphi(t)+P(x, y)}=1-N(x, y, t)
$$

is a fuzzy metric for $*$. Then, by Proposition $1.4,\left(\mathrm{X}, 1-\mathrm{N}, \mathrm{N}, *, *^{\prime}\right)$ is an IFMS. By hypothesis, $\mathrm{K}+\mathrm{P} \leqslant 1$ and then $P \leqslant 1-K$ and hence $M \leqslant 1-N$, and consequently, by Lemma $3.1\left(X, M, N, *, *^{\prime}\right)$ is an IFMS.

\section{Strong intuitionistic fuzzy metric spaces}

Definition $4.1([2])$. Let $(X, M, N, *, \diamond)$ be an IFMS. The IFM $(M, N)$ is said to be strong if it satisfies for each $x, y, z \in X$ and each $t>0$,

$$
\begin{aligned}
& M(x, z, t) \geqslant M(x, y, t) * M(y, z, t) \\
& N(x, z, t) \leqslant N(x, y, t) \diamond N(y, z, t) .
\end{aligned}
$$

By this definition, Proposition 1.10, and (ii)-(iii) of Proposition 1.1, we obtain the following proposition.

Proposition 4.2. $(X, M, N, *, \diamond)$ is a strong IFMS if and only if $(M, *)$ and $\left(1-N, \diamond^{\prime}\right)$ are strong fuzzy metrics on $\mathrm{X}$ and $\mathrm{M}+\mathrm{N} \leqslant 1$.

By (ii)-(iii) of Proposition 1.1 we obtain the following proposition.

Proposition 4.3. An IFMS $\left(X, M, 1-M, *, *^{\prime}\right)$ is strong if and only if $(X, M, *)$ is a strong fuzzy metric space.

Remark 4.4 (Compare with [2, Example 2.2 and Remark 2.3]). The extension of Remark 2.2 and Proposition 2.3 to an IFMS $(X, M, N, *, \diamond)$ is obvious since $(X, M, *)$ and $\left(X, 1-N, \diamond^{\prime}\right)$ are fuzzy metric spaces. 
Remark 4.5 (Compare with [2, Example 2.9]). In [13, Example 9 (c)] the authors stated that $\left(M_{1}, *\right)$ is a strong fuzzy metric on $\mathbb{R}^{+}$where $*$ is the usual product and $M_{1}(x, y, t)=\frac{\min \{x, y\}+t}{\max \{x, y\}+t}$.

Then it is easy to conclude that this assertion is also true for $(M, *)$ where $M(x, y, t)=\frac{\min \{x, y\}+\varphi(t)}{\max \{x, y\}+\varphi(t)}$ and $\varphi(t): \mathbb{R}^{+} \rightarrow \mathbb{R}^{+}$is a non-decreasing continuous function.

Indeed,

$$
\begin{aligned}
M(x, z, t)=\frac{\min \{x, z\}+\varphi(t)}{\max \{x, z\}+\varphi(t)} & =M_{1}(x, z, \varphi(t)) \\
& \geqslant M_{1}(x, y, \varphi(t)) \cdot M_{1}(y, z, \varphi(t)) \\
& =\frac{\min \{x, y\}+\varphi(t)}{\max \{x, y\}+\varphi(t)} \cdot \frac{\min \{y, z\}+\varphi(t)}{\max \{y, z\}+\varphi(t)}=M(x, y, t) \cdot M(y, z, t) .
\end{aligned}
$$

Consequently, by Proposition $1.4,\left(\mathbb{R}^{+}, M, 1-M, *, *^{\prime}\right)$ is a strong IFMS.

Remark 4.6 (Compare with [2, Example 2.12]). Let $\left(\mathrm{X}, \mathrm{K}, \mathrm{P}, *, *^{\prime}\right)$ be a stationary IFMS, where $*$ is the usual product. Let $M, N$ be the fuzzy sets on $X^{2} \times \mathbb{R}^{+}$defined by $M(x, y, t)=\frac{t+K(x, y)}{t+1}$ and $N(x, y)=,\frac{P(x, y)}{t+1}$. By [13, Example $9(\mathrm{e})],(\mathrm{M}, *)$ is a strong fuzzy metric. By Proposition $1.10,(1-\mathrm{P}, *)$ is a (strong) fuzzy metric. Then, again by [13, Example $9(\mathrm{e})],\left(\frac{\mathrm{t}+1-\mathrm{P}(x, y)}{\mathrm{t}+1}, *\right)$ is a strong fuzzy metric.

Then, by Proposition 1.4, $\left(\frac{\mathrm{t}+1-\mathrm{P}(\mathrm{x}, \mathrm{y})}{\mathrm{t}+1}, 1-\frac{\mathrm{t}+(1-\mathrm{P}(\mathrm{x}, \mathrm{y}))}{\mathrm{t}+1}, *, *^{\prime}\right)$ is a strong IFM. Finally, by Lemma 3.1 and Proposition $4.2,\left(\mathrm{M}, \mathrm{N}, *, *^{\prime}\right)$ is a strong IFM, since $\mathrm{K} \leqslant 1-\mathrm{P}$.

Remark 4.7. From [13, Example 9 (f),(g),(h),(i)], and Proposition 1.4, we obtain that Examples 2.13, 2.14, and 2.15 in [2] are obvious.

Remark 4.8. The extension of Proposition 2.5 to the intuitionistic setting has been made in [2, Proposition 2.20]. Now, it is an immediate consequence of Proposition 1.10 and Proposition 1.11 (i).

Remark 4.9. Examples 2.21 and 2.22 of [2] are immediate extensions of [13, Example 14 (a) and (b)], to the intuitionistic setting.

The following definition is due to Park [12].

Definition 4.10. Let $(X, M, N, *, \diamond)$ be an IFMS. Then:

(i) a sequence $\left\{x_{n}\right\}$ is said to be Cauchy if for each $\varepsilon>0$ and each $t>0$, there exists $n_{0} \in \mathbb{N}$ such that $M\left(x_{m}, x_{n}, t\right)>1-\varepsilon$ and $N\left(x_{m}, x_{n}, t\right)<\varepsilon$ for all $m, n \geqslant n_{0}$;

(ii) $(X, M, N, *, \diamond)$ is called complete if every Cauchy sequence in $X$ is convergent with respect to $\tau_{(M, N)}$.

Now, due to the axiom (IFM-1) it is obvious that a sequence $\left\{x_{n}\right\}$ is Cauchy in $(X, M, N, *, \diamond)$ if and only if $\left\{x_{n}\right\}$ is Cauchy in the fuzzy metric space $(X, M, *)$. Then, recalling that $\tau_{(M, N)}=\tau_{M}$, we have that $(X, M, N, *, \diamond)$ is complete if and only if $(X, M, *)$ is complete. In consequence, the formulation in the intuitionistic setting of Proposition 2.23 and Corollary 2.24 of [2] as extensions of Proposition 2.6 and Corollary 2.7, respectively, have, really, no sense. Further, the converse of [2, Corollary 2.24] is not a consequence of the fact that $\tau_{M}\left(=\tau_{(M, N)}\right)=\bigvee\left\{\tau_{M_{t}}: t>0\right\}$. Here we write a proof as following.

Suppose that $\left\{x_{n}\right\}$ is a Cauchy sequence in the strong fuzzy metric space $(X, M, *)$ and that $\left(X, M_{t}, *\right)$ is complete for each $t>0$. Then, by Proposition 2.6, $\left\{x_{n}\right\}$ is Cauchy in $\left(X, M_{t}, *\right)$ for all $t>0$. Then, for each $t>0$ there exists $x_{t} \in X$ such that $\left\{x_{n}\right\}$ converges to $x_{t}$ in $\left(X, M_{t}, *\right)$. We will see that $x_{t}=x_{0}$ for all $t>0$. Indeed, if $t_{1}<t_{2}$ then $\lim _{n} M_{t_{1}}\left(x_{n}, x_{t_{1}}\right)=1$ implies, by Proposition 2.5, that $\lim _{n} M_{t_{2}}\left(x_{n}, x_{t_{1}}\right)=1$ and so $\left\{x_{n}\right\}$ converges also to $x_{t_{1}}$ in $\left(X, M_{t_{2}}, *\right)$, that is $x_{t_{1}}=x_{t_{2}}\left(=x_{0}\right)$, since the limit is unique. Then $\left\{x_{n}\right\}$ converges in $\tau_{M_{t}}$ to $x_{0}$ for all $t>0$. Hence, $\lim _{n} M_{t}\left(x_{n}, x_{0}\right)=1$ for all $t>0$ and, consequently, $\lim _{n} M\left(x_{n}, x_{0}, t\right)=1$ for all $t>0$ that is $\left\{x_{n}\right\}$ converges to $x_{0}$ in $(X, M, *)$ and then $(X, M, *)$ is complete. 


\section{Acknowledgment}

Valentín Gregori is supported by the Spanish Ministry of Economy and Competitiveness under Grant MTM2015-64373-P and Almanzor Sapena is supported by Generalitat Valencia under Grant $\mathrm{AICO} / 2017 / 059$.

\section{References}

[1] K. T. Atanassov, Intuitionistic fuzzy sets, Fuzzy Sets and Systems, 20 (1986), 87-96. 1

[2] H. Efe, E. Yigit, On strong intuitionistic fuzzy metric spaces, J. Nonlinear Sci. Appl., 9 (2016), 4016-4038. 1, 1.4, 1, 1.9, 3.3, 3.4, 3.5, 3.6, 4.1, 4.4, 4.5, 4.6, 4.7, 4.8, 4.9, 4

[3] V. Gregori, J.-J. Miñana, S. Morillas, A. Sapena, Characterizing a class of completable fuzzy metric spaces, Topology Appl., 203 (2016), 3-11. 1

[4] V. Gregori, S. Morillas, A. Sapena, On a class of completable fuzzy metric spaces, Fuzzy Sets and Systems, 161 (2010), 2193-2205. 1, 2

[5] V. Gregori, S. Morillas, A. Sapena, Examples of fuzzy metrics and applications, Fuzzy Sets and Systems, 170 (2011), 95-111. 1, 3.3, 3.5, 3.6

[6] V. Gregori, S. Romaguera, Some properties of fuzzy metric spaces, Fuzzy Sets and Systems, 115 (2000), 485-489. 1

[7] V. Gregori, S. Romaguera, Characterizing completable fuzzy metric spaces, Fuzzy Sets and Systems, 144 (2004), 411420. 1.9

[8] V. Gregori, S. Romaguera, P. Veeramani, A note on intuitionistic fuzzy metric spaces, Chaos Solitons Fractals, 28 (2006), 902-905. 1, 1.4, 1, 1.10

[9] A. George, P. Veeramani, On some results in fuzzy metric spaces, Fuzzy Sets and Systems, 64 (1994), 395-399. 1, 1.3, 1

[10] A. George, P. Veeramani, Some theorems in fuzzy metric spaces, J. Fuzzy Math., 3 (1995), 933-940. 1

[11] I. Kramosil, J. Michálek, Fuzzy metric and statistical metric spaces, Kybernetika, 11 (1975), 336-344. 1

[12] J. H. Park, Intuitionistic fuzzy metric spaces, Chaos Solitons \& Fractals, 22 (2004), 1039-1046. 1, 1, 1, 1.10, 4

[13] A. Sapena, S. Morillas, On strong fuzzy metric spaces, Proc. Workshop Appl. Topology, 9 (2009), 135-141. 1, 2, 2.2, $2.4,2.5,2.6,2.7,4.5,4.6,4.7,4.9$

[14] B. Schweizer, A. Sklar, Statistical metric spaces, Pacific J. Math., 10 (1960), 313-334. 1 